\title{
Continuo de conductas alimentarias de riesgo en adolescentes de México
}

\author{
Martha Betzaida Altamirano Martínez, ${ }^{1}$ Barbara Vizmanos Lamotte ${ }^{1}$ \\ y Claudia Unikel Santoncini²
}

Forma de citar

Altamirano Martínez MB, Vizmanos Lamotte B, Unikel Santoncini C. Continuo de conductas alimentarias de riesgo en adolescentes de México. Rev Panam Salud Publica. 2011;30(5);401-7.

RESUMEN Objetivo. Determinar las relaciones existentes entre las variables autoestima (AU), insatisfacción corporal (IC) e indice de masa corporal (IMC) en un continuo de conductas alimentarias de riesgo (CAR) que abarque desde la normalidad hasta el riesgo de trastornos de conducta alimentaria (TCA), facilitando así la detección temprana de adolescentes con manifestaciones de TCA y el grado en que influyen dichas variables.

Métodos. Se seleccionaron 1982 mujeres de 15 a 19 años de edad, de acuerdo con un muestreo aleatorio estratificado. Se midió la AU (escala de Pope, McHale y Craighead), la IC ("Escala análoga de figuras corporales" de Stunkard) y el IMC. Se administró un cuestionario breve de CAR. Se definieron tres categorías de CAR: sin riesgo, riesgo moderado y riesgo alto. Se realizó la prueba de regresión multinomial.

Resultados. Se encontró una relación directa entre el riesgo de CAR y la IC, con diferencias significativas entre grupos ( $\mathrm{P}<0,001)$ : sin riesgo (83,6\% de la muestra) $54,1 \%$ presentaron IC; con riesgo moderado (11,9\% de la muestra) $84,8 \%$ presentaron IC, $y$ de riesgo alto (4,5\% de la muestra) 89,9\% presentaron IC. En AU e IMC solo hubo diferencias significativas entre el grupo sin riesgo y cada uno de los grupos de riesgo. La AU participó en la predicción de CAR en todos los modelos de regresión evaluados, mientras que el IMC no lo hizo.

Conclusiones. Es perentorio identificar adolescentes en riesgo moderado de CAR que revelen características prodrómicas de TCA, como baja AU e IC, las cuales, cuando se presentan de forma conjunta, pueden mediar un efecto negativo del IMC sobre las CAR.

Palabras clave Conducta alimentaria; salud del adolescente; autoestima; índice de masa corporal; imagen corporal; factores de riesgo; obesidad; sobrepeso; México.

Las conductas alimentarias "de riesgo" (CAR) — también denominadas "disfuncionales" — son manifestaciones simila-

\footnotetext{
1 Cuerpo Académico 454, Laboratorio de Evaluación del Estado Nutricio, Departamento de Reproducción Humana, Crecimiento y Desarrollo Infantil, Centro Universitario de Ciencias de la Salud, División de Disciplinas Clínicas, Universidad de Guadalajara. La correspondencia se debe dirigir a Claudia Unikel Santoncini. Correo electrónico: unikels@imp.edu.mx

2 Dirección de Investigaciones Epidemiológicas y Psicosociales del Instituto Nacional de Psiquiatría

"Ramón de la Fuente Muñiz", D.F., México.
}

res a los trastornos de conducta alimentaria (TCA) pero se dan con menor frecuencia e intensidad (1). Según la teoría del continuo propuesta por Nylander en 1971, las CAR se encuentran en la parte intermedia de dicho continuo: en un extremo está la conducta alimentaria normal y en el otro los TCA (2). Las CAR incluyen atracones, seguimiento de dietas restrictivas, abuso de laxantes, diuréticos o anfetaminas, exceso de ejercicio, vómito autoinducido y práctica de ayunos, todos dirigidos a controlar o reducir el peso corporal (1).

Desde esta perspectiva, las personas con alimentación restringida o "dietantes crónicos" presentan un menor grado de psicopatología que quienes padecen TCA —comienzan voluntariamente con conductas de riesgo en pequeña escala y evolucionan hasta llegar a la pérdida de control y el síndrome completo $(3,4)$.

En estudios acerca de las causas de CAR y de TCA se han identificado facto- 
res de riesgo biológicos (p. ej. alteraciones genéticas y neurológicas, género [femenino] y etapa de la vida [adolescencia]) y psicológicos y sociales (p. ej. sensación de fracaso, estrés, ansiedad, depresión, abuso físico o sexual, problemas familiares, comparación social, burlas de pares y familiares, baja autoestima [AU] e insatisfacción corporal [IC]) (512). Tal grado de multicausalidad en estos trastornos obliga a considerar una perspectiva biopsicosocial para su estudio (13).

Entre los factores de mayor impacto reportados en el desarrollo de CAR se encuentran la baja AU y la IC, esta última con gran predominio durante la adolescencia como resultado de diversos cambios físicos y la relevante influencia de amigos y pares, así como de la presión social que promueve la delgadez (6, 13-15). La fuente de la IC radica particularmente en la discrepancia que suele darse entre la figura corporal real y la considerada ideal, sobre todo cuando tiene lugar en personas con deficiencias yoicas y se manifiesta en baja AU (16). La IC conduce a CAR como medio para alcanzar el ideal de delgadez promovido socialmente. La relación entre ambas variables se ha analizado en programas de prevención orientados a elevar la $\mathrm{AU}$, comprobándose que a medida que esta variable aumenta también lo hace la satisfacción con la imagen corporal (17).

Una de las deficiencias en los métodos actuales para la detección de adolescentes en riesgo de desarrollar un TCA estriba en que aplican cuestionarios que identifican, mediante un punto de corte, casos con alto riesgo pero no incluyen medidas intermedias que ayuden a descubrir casos con síntomas menos intensos que permitirían prevenir la enfermedad en una etapa más temprana.

El objetivo del presente trabajo es determinar las relaciones existentes entre las variables $A U, I C$ e índice de masa corporal (IMC) en un continuo de CAR que abarque desde la normalidad hasta el riesgo de presentar TCA, facilitando así la detección oportuna de adolescentes con manifestaciones de TCA y el grado en que influyen dichas variables.

\section{MATERIALES Y MÉTODOS}

\section{La muestra}

Se realizó un estudio transversal con un muestreo aleatorio estratificado por nivel de marginación, el cual mide el impacto global de las carencias que padece la población en dos niveles (bajo y alto) y la intensidad de migración de las localidades donde tuvo lugar el proyecto en tres niveles (bajo, medio y alto), con un resultado final de seis estratos $(18,19)$. La muestra estuvo compuesta por mujeres inscritas en los planteles escolares del sector público de nivel medio superior en el estado de México y registradas por la Secretaría de Educación Pública durante el ciclo lectivo 2005-2006. Las escuelas participantes fueron seleccionadas de acuerdo con el número de mujeres inscritas, a partir de un total de 661 escuelas con 174419 alumnas, estableciéndose una cifra proporcional para cada estrato. Mediante este procedimiento fueron elegidas 11 escuelas pertenecientes a 11 municipios de ese estado. La muestra quedó constituida por 2358 mujeres, aunque de este número solo se incluyeron las que contestaron en su totalidad los tres instrumentos aplicados y que además fueron evaluadas antropométricamente, resultando entonces un total de 1982 mujeres con una media de edad de 16,3 años (desviación estándar $[D E]=1,0)$ y un rango de 15 a 19 años.

Una vez seleccionados los planteles, se solicitó el consentimiento escrito de las autoridades escolares, quienes a su vez informaron a los padres acerca de la participación de sus hijas en el estudio, que consistiría en responder un cuestionario de autorrespuesta en las aulas y en permitir que se les midan el peso y la estatura. Se encuestó a todas las estudiantes seleccionadas que acudieron el día de la evaluación, con excepción de un plantel donde la mitad de las aulas bastó para completar la cuota necesaria para el estrato correspondiente.

Las participantes fueron invitadas a colaborar voluntariamente en el estudio, informándoseles acerca del objetivo y los procedimientos de su participación, así como su carácter anónimo y confidencial. El presente trabajo forma parte de un proyecto que fue aprobado por el Comité de Ética del Instituto Nacional de Psiquiatría "Ramón de la Fuente Muñiz". Fue también evaluado por el Consejo Nacional de Ciencia y Tecnología (Conacyt), que además lo financió.

\section{Instrumentos}

Los instrumentos utilizados en el estudio fueron validados previamente en una muestra similar de estudiantes en el estado de México (20). La evaluación de la AU se llevó a cabo mediante la "Escala de autoestima" de Pope, McHale y Craighead, validada en México por Caso y por Unikel y Gómez (21-23). Esta escala consta de nueve preguntas tipo Likert, con cuatro opciones de respuesta (nunca o casi nunca, algunas veces, frecuentemente y muy frecuentemente). Cada opción de respuesta tiene un valor de uno a cuatro puntos; una mayor puntuación se asocia a más baja AU. La escala obtuvo una confiabilidad de 0,83 y se organiza en dos factores que explican $59,67 \%$ de la varianza total: uno contiene reactivos en sentido positivo, como "Me gusta como soy", y otro en sentido negativo, como "Tengo una mala opinión de mí misma".

La IC se midió con la versión adaptada y validada en México por Acosta García y Gómez Peresmitré de la "Escala análoga de figuras corporales", originalmente desarrollada por Stunkard, Sorensen y Schulsinger $(24,25)$. Esta escala muestra una confiabilidad test-retest de 0,89 y una correlación significativa de 0,62 respecto a la subescala de insatisfacción corporal del EAT-26 (26). Consta de nueve siluetas que cubren un continuo de peso corporal, en el cual se incluyen figuras muy delgadas, delgadas, normales, gruesas y muy gruesas. Estas imágenes impresas - puntuadas de uno a nueve, de la más delgada a la más gruesa- se utilizan en dos ocasiones: en la primera se invita al sujeto a elegir la silueta que mejor representa su figura corporal actual, y después a elegir la silueta que más le gustaría tener (figura ideal). Luego se obtiene la diferencia en la puntuación entre ambas (figura actual-figura ideal), con tres resultados posibles: i) diferencia nula (indica satisfacción con la imagen corporal), ii) diferencia positiva (indica insatisfacción en cuanto al deseo de una figura más delgada) y iii) diferencia negativa (indica insatisfacción en cuanto al deseo de una figura más gruesa) (25).

Las CAR se midieron con el "Cuestionario breve de conductas alimentarias de riesgo" (CBCAR), basado en los criterios para el diagnóstico de TCA del "Manual diagnóstico y estadístico de los trastornos mentales" (DSM [IV], por sus siglas en inglés). Este cuestionario fue diseñado ex profeso para detectar CAR para la Encuesta sobre la Prevalencia del Consumo de Drogas y Alcohol en la Pobla- 
ción Estudiantil del Distrito Federal de $1997(27,28)$. Esta herramienta, validada en pacientes con diagnóstico de TCA en tratamiento y una muestra comunitaria $\mathrm{y}$, posteriormente, con una población similar a la del presente estudio, obtuvo una confiabilidad de 0,81 y se organiza en tres factores que explican $62,96 \%$ de la varianza: conductas restrictivas, purgativas y atracones (1).

El cuestionario incluye una pregunta sobre la preocupación por engordar; dos sobre la práctica de atracones con sensación de falta de control; cuatro sobre conductas de tipo restrictivo (p. ej. dieta, ejercicio, ayunos o pastillas para adelgazar), y tres sobre conductas de tipo purgativo (p. ej. vómito autoinducido, laxantes o diuréticos). Se califica con cuatro opciones de respuesta: $0=$ nunca o casi nunca, 1 = algunas veces, 2 = frecuentemente (dos veces a la semana) y 3 = muy frecuentemente (más de dos veces a la semana). Los autores propusieron un punto de corte mayor de 10 para identificar personas en riesgo o posibles casos de TCA, el cual fue determinado mediante los valores de especificidad y sensibilidad de la escala (1).

Para analizar las relaciones de las variables independientes en el continuo de CAR, se estableció un punto de corte adicional al planteado en el CBCAR, denominado "riesgo moderado" y definido con un valor superior o igual a la media más una DE de la calificación obtenida en el CBCAR para la población estudiada $(\geq$ media+1DE). De esta manera quedó una clasificación en tres niveles: i) sin riesgo (0 a 6 puntos), ii) riesgo moderado (7 a 10 puntos) y iii) riesgo alto (>10 puntos).

El IMC se calculó según la ecuación: peso $(\mathrm{kg})$ dividido por estatura $(\mathrm{m})$ al cuadrado. Las categorías de IMC consi- deradas fueron las del Grupo Internacional de Trabajo sobre Obesidad (IOTF, por sus siglas en inglés) para niños y adolescentes (29).

\section{Análisis estadístico}

Los datos, analizados con los programas SPSS para Windows versión 13 (SPSS, 2004) y SAS (The SAS Institute, 2003), se presentan como porcentajes para las variables nominales $\mathrm{u}$ ordinales, y como medias y DE para las variables continuas. En virtud de que las variables no mostraron una distribución normal, se utilizaron pruebas no paramétricas (Kruskal- Wallis y U de Mann Whitney) para obtener las diferencias entre los grupos de CAR en AU, IC e IMC.

Para establecer el modelo de interacción entre las variables independientes (AU, IC e IMC) en los tres grupos de CAR, se realizó un análisis de regresión múltiple utilizando la variable CAR en forma continua y, posteriormente, de regresión logística multinomial con dicha variable en los niveles de riesgo propuestos. Para estas pruebas se consideró un valor de significancia $P<0,05$.

\section{RESULTADOS}

En el cuadro 1 se presentan los datos descriptivos del estudio. Con respecto a la imagen corporal (datos no mostrados), poco más de la mitad de las adolescentes $(63,8 \%, n=1264)$ se mostraron insatisfechas con su imagen corporal: $53 \%(n=$ $1050)$ deseaban una figura más delgada y $10,8 \%(n=214)$, una figura más gruesa. Solo $36,2 \%(n=718)$ demostraron estar satisfechas.

Aunque desear una figura más gruesa es parte de la IC, las adolescentes en esta

CUADRO 1. Características de una muestra conformada por estudiantes mujeres de escuelas públicas para detectar conductas alimentarias de riesgo, México, 2007 $(n=1768)$

\begin{tabular}{lcccc}
\hline \multicolumn{1}{c}{ Indicador } & Media & $\mathrm{DE}^{\mathrm{a}}$ & Mínimo & Máximo \\
\hline Edad (años) & 16,3 & 1,0 & 15,0 & 19,0 \\
Peso (kg) & 57,5 & 10,2 & 31,6 & 112,0 \\
Talla (m) & 1,55 & 0,06 & 1,15 & 1,77 \\
Índice de masa corporal & 23,7 & 3,7 & 17,0 & 41,8 \\
Insatisfacción corporal & 1,0 & 1,1 & 0,0 & 7,0 \\
Autoestima & 16,1 & 5,1 & 9,0 & 34,0 \\
Conductas alimentarias de riesgo & 3,7 & 3,4 & 0,0 & 27,0 \\
\hline
\end{tabular}

Fuente: elaboración de los autores.

Nota: Escalas utilizadas. Índice de masa corporal: peso/talla al cuadrado; Insatisfacción corporal: Escala análoga de figuras corporales; Autoestima: Escala de autoestima de Pope; Conductas alimentarias de riesgo: Cuestionario breve de conductas alimentarias de riesgo.

a DE: desviación estándar. situación mostraron una tendencia inversa a lo que se espera en los TCA: de acuerdo con un análisis de correlación aplicado exclusivamente en estas adolescentes, no se encontró una correlación significativa entre las variables IC y CAR $(R=-0,03, P>0,05)$. Por lo anterior, y debido a que eran pocas $(10,8 \%, n=214)$, estas participantes fueron excluidas en los análisis subsecuentes. La población restante $(n=1768)$ se distribuyó en $40,6 \%$ $(n=718)$ de adolescentes satisfechas con su imagen corporal y 59,2\% $(n=1050)$ que deseaban una figura más delgada.

Respecto al IMC, la distribución del estado nutricio según los puntos de corte del IOTF (29) fue de $1,3 \%$ en bajo peso $(n=23), 65,9 \%$ en normal $(n=1165)$, $25,4 \%$ en sobrepeso $(n=449)$ y $7,4 \%$ en obesidad $(n=131)$. En cuanto al CBCAR, la distribución porcentual para cada una de las categorías propuestas fue de $83,6 \%$ para el grupo sin riesgo $(n=$ $1478), 11,9 \%$ para el grupo con riesgo moderado $(n=211)$ y $4,5 \%$ para el grupo con riesgo alto $(n=79)$.

Al comparar las puntuaciones de IC, AU e IMC en los tres niveles de CAR, se advirtieron diferencias significativas en todos los casos $(P<0,001)$ (cuadro 2$)$. En el grupo de alto riesgo de CAR se observó una mayor proporción de IC $(89,9 \%, n=71)\left(X^{2}=104,1, P<0,001\right)$ en comparación con los grupos de menor riesgo de CAR (figura 1). La IC mostró diferencias estadísticamente significativas $(P<0,001)$ entre los tres niveles de riesgo de CAR.

La prueba de Mann-Whitney en pares de grupos mostró que, para AU e IMC, solo hubo diferencias significativas entre las adolescentes sin riesgo de CAR y aquellas con riesgo moderado y alto $(P<0,001)$. No se encontraron diferencias significativas en el análisis entre las adolescentes con riesgo moderado y alto, a pesar de que estas últimas presentaron mayor puntuación en las escalas de AU e IMC.

\section{Modelo de interacción de las variables independientes}

Se realizó una regresión múltiple para valorar la contribución de IC, AU e IMC en la varianza de CAR, con resultado significativo únicamente en las dos primeras variables $\left(R^{2}=0,264, P<\right.$ 0,001). Sin embargo, cuando se incluyó la interacción de AU e IC, dicha interacción mostró un cambio importante en $\mathrm{R}^{2}$ de 0,017 para la varianza de CAR, lo 
CUADRO 2. Diferencias en la puntuación de autoestima, insatisfacción corporal e índice de masa corporal de acuerdo a los niveles de conductas alimentarias de riesgo, México, 2007 ( $n=1768$ )

\begin{tabular}{lcccc}
\hline & \multicolumn{4}{c}{$\begin{array}{c}\text { Niveles de riesgo } \\
\text { Mediana (P25-P75) }\end{array}$} \\
\cline { 2 - 4 } Variable independiente & $\begin{array}{c}\text { Sin riesgo } \\
(n=1478)\end{array}$ & $\begin{array}{c}\text { Riesgo moderado } \\
(n=211)\end{array}$ & $\begin{array}{c}\text { Riesgo alto } \\
(n=79)\end{array}$ & $P^{\mathrm{b}}$ \\
\hline Insatisfacción corporal & $1(0-1)$ & $1(1-2)$ & $2(1-3)$ & $<0,001^{\mathrm{c}}$ \\
Autoestima & $15(12-19)$ & $20(16-23)$ & $20(17-24)$ & $<0,001^{\text {d }}$ \\
Índice de masa corporal & $22,7(20,9-25,5)$ & $23,6(22,1-26,5)$ & $24,4(22,1-27,2)$ & $<0,001^{\text {d }}$
\end{tabular}

Fuente: elaboración de los autores.

a $P$ : percentil.

b Según la prueba de Kruskal-Wallis.

c $P<0,001$ según la prueba de Mann-Whitney por pares: sin riesgo vs. riesgo moderado; sin riesgo vs. riesgo alto; riesgo moderado vs. riesgo alto.

d $P<0,001$ según la prueba de Mann-Whitney por pares: sin riesgo vs. riesgo moderado; sin riesgo vs. riesgo alto.

que dio lugar a una $\mathrm{R}^{2}$ total del modelo con las tres variables de $0,28(P<0,001)$; no obstante, se perdió la significancia de IC y el IMC se mantuvo igual, aunque con mayor fuerza en el modelo (cuadro 3).

Finalmente, se elaboró un modelo utilizando una regresión multinomial. Debido a que la interacción entre IC y AU no fue significativa, en este caso se incluyó la IC en forma dicotómica y se tomó como grupo de referencia a las adolescentes sin riesgo de CAR. Los resultados indicaron que, ante IC, hay un riesgo de CAR de 2,0 (intervalo de confianza de 95\% [IC95\%]: 1,3-3,0) para las jóvenes del grupo de alto riesgo, y de 1,8 en las adolescentes en riesgo moderado (IC95\%: 1,5-2,2). Asimismo, ante una mayor puntuación en $\mathrm{AU}$, el riesgo de CAR fue mayor en el grupo en riesgo alto (razón de probabilidades $[R P]=1,2$; IC $95 \%$ : $1,2-1,3)$ en comparación con el de riesgo moderado ( $R P=1,1$; IC95\%: 1,1-1,2), con diferencias significativas respecto a las adolescentes sin riesgo de CAR $(P<$ $0,001)$ en todos los casos. No se encontraron diferencias significativas en el IMC, ni en las adolescentes con riesgo moderado, ni tampoco en las de riesgo alto en relación con las adolescentes sin riesgo (cuadro 4).

\section{DISCUSIÓN}

Los resultados del presente trabajo mostraron que i) la IC y la AU contribuyen en la varianza de CAR; ii) la interacción IC-AU aumenta la fuerza del IMC para afectar las CAR, aunque no de forma significativa, y iii) cuando se analizan las CAR en niveles de riesgo, se observa un efecto significativo de la IC y de la AU, pero no del IMC.

Asimismo, a mayor psicopatología en IC, mayor es la probabilidad de cambiar de nivel de riesgo de CAR. Este hallazgo coincide con lo observado por
Griffiths y McCabe en una evaluación de 111 mujeres en la adolescencia temprana (11 a 13 años) y reafirma lo declarado por otros autores que consideran la variable IC como uno de los factores de mayor peso para el desarrollo de TCA (30-32). Sin embargo, en el presente estudio se consideran las CAR en niveles de riesgo, en donde la IC se posiciona como un factor importante para identificar a las adolescentes en riesgo moderado, como una estrategia de prevención secundaria.

No hubo diferencia significativa en AU e IMC entre las adolescentes en riesgo moderado y alto, pero se encontró mayor patología en ambas con respecto a las adolescentes sin riesgo. Estos resultados concuerdan con otros estudios transversales que además correlacionan la $\mathrm{AU}$ con aspectos restrictivos y purgativos, mientras que al IMC solo lo asocian con conductas restrictivas $(30,33)$.

El hecho de que en la regresión múltiple el IMC tuviera más fuerza, aunque no de manera relevante, y que la propia IC dejara de ser significativa al añadir la interacción AU-IC, muestra la influencia que puede ejercer la $\mathrm{AU}$ como un factor mediador en el efecto de estas variables independientes, ya que fue la única que mantuvo una participación constante en la predicción de CAR en todos los modelos de regresión evaluados. Este efecto mediador ya se ha referido en otros trabajos - transversales y longitudinalesrealizados en poblaciones diversas que van desde adolescentes hasta adultos jóvenes $(30,33-36)$. No obstante, dichos trabajos no consideraron la interacción AU-IC, lo que podría explicar que en sus resultados el IMC mantiene su efecto sobre las CAR, lo cual podría reflejar también que el IMC influye más directamente en la IC que en las CAR.

De allí que la manifestación de baja AU en adolescentes con IC podría mediar el desarrollo de CAR, sobre todo a mayor IMC, pero tal relación tendría que valorarse en una muestra con mayor grado de obesidad. Contrariamente, es probable que al no presentar IC combinada con baja AU, el IMC podría no influir en el riesgo de CAR, aunque el estudio longitudinal de Shaw y colaboradores no sustenta la relación mediadora de la AU con CAR (37). Se requieren pues más investigaciones que contemplen este continuo de CAR y su asociación con AU, IC e IMC en evaluaciones seriadas a largo plazo. 
CUADRO 3. Predicción de conductas alimentarias de riesgo por insatisfacción corporal (IC), autoestima (AU), índice de masa corporal y la interacción entre insatisfacción corporal y autoestima, México, $2007(n=1768)$

\begin{tabular}{lcccc}
\hline \multirow{2}{*}{ Variable } & \multicolumn{3}{c}{ Coeficientes beta } \\
\cline { 2 - 5 } & Paso 1 & Paso 2 & Paso 3 & Paso 4 \\
\hline Insatisfacción corporal & $0,455^{\mathrm{a}}$ & $0,355^{\mathrm{a}}$ & $0,348^{\mathrm{a}}$ & $-0,071$ \\
Autoestima & $\ldots . \mathrm{b}$ & $0,261^{\mathrm{a}}$ & $0,262^{\mathrm{a}}$ & $0,138^{\mathrm{a}}$ \\
Índice de masa corporal & $\ldots$. & $\ldots$ & 0,013 & 0,031 \\
Interacción IC x AU & $\ldots$. & $\ldots$ & $\ldots, 490^{\mathrm{a}}$ \\
$\mathrm{R}^{2}$ ajustada & 0,206 & $0,264^{\mathrm{a}}$ & $0,264^{\mathrm{a}}$ & $0,280^{\mathrm{a}}$ \\
Cambios en $\mathrm{R}^{2}$ & $0,206^{\mathrm{a}}$ & $0,058^{\mathrm{a}}$ & 0,000 & $0,017^{\mathrm{a}}$ \\
\hline
\end{tabular}

Fuente: elaboración de los autores.

Nota: Regresión lineal múltiple sobre la varianza de conductas alimentarias de riesgo por pasos.

a $P<0,001$.

b (...): Sin datos.

CUADRO 4. Regresión multinomial en los tres niveles de conducta alimentaria de riesgo según insatisfacción corporal, autoestima e índice de masa corporal, México, 2007 ( $n=1768)$

\begin{tabular}{|c|c|c|c|}
\hline \multirow[b]{2}{*}{ Variable } & \multicolumn{3}{|c|}{$\begin{array}{c}\text { Niveles de CAR } \\
\text { Razón de probabilidades (IC95\%) }\end{array}$} \\
\hline & Sin riesgo & Riesgo moderado & Riesgo alto \\
\hline Insatisfacción corporal & 1 & $1,8(1,5-2,2)^{a}$ & $2,0(1,3-3,0)^{a}$ \\
\hline Autoestima & 1 & $1,1(1,1-1,2)^{a}$ & $1,2(1,2-1,3)^{a}$ \\
\hline Índice de masa corporal & 1 & $1,0(1,0-1,0)$ & $1,1(1,0-1,1)$ \\
\hline
\end{tabular}

Los hallazgos del presente estudio respaldan la teoría del continuo, al mostrar diferencias psicopatológicas respecto a IC y AU en las adolescentes sin riesgo y aquellas en riesgo moderado de CAR (38-41), mientras que el IMC per se no necesariamente implicaría un riesgo. La asociación AU-IC podría favorecer que las adolescentes se muevan de un nivel de CAR a otro.

Otra de las aportaciones de esta investigación es que brinda una herramienta breve para detectar sujetos con riesgo de CAR en una etapa temprana, facilitando medidas de prevención. Si bien en México ya se contaba con instrumentos que permitían identificar sujetos con riesgo alto (p. ej. el CBCAR y el EAT-40, Eating Attitude Test), en línea con lo señalado en la literatura sobre prevención de TCA y CAR se recomienda que las intervenciones preventivas sean hechas en etapas tempranas de la manifestación de los sín- tomas para aumentar su efectividad (42). Esta es una medida fundamental en la realidad actual mexicana, donde 32,5\% de los adolescentes padecen sobrepeso $\mathrm{u}$ obesidad, factores que podrían ser de riesgo para el desarrollo de CAR y TCA en personas vulnerables (43-45).

Una de las limitaciones de este estudio radica en que, aun cuando hace posible evaluar la fuerza de asociación entre las variables en juego, no permite identificar una asociación causal. Asimismo, el instrumento aplicado de AU —validado y utilizado en otros estudios de conducta alimentaria- no ha sido empleado todavía en estudios internacionales, factor que podría dificultar la comparación de los resultados con estudios que usan otras escalas $(22,23,37,39)$. Por otra parte, en esta investigación no se consideraron adolescentes de escuelas privadas, quienes según otros trabajos registran mayor prevalencia de TCA que los de escuelas públicas (46). Al no incluir hombres, tampoco se develó el comportamiento de las variables estudiadas en este grupo de población ni se pudo hacer un análisis de género.

Finalmente, dado que el estudio no se aplicó a una muestra de pacientes con TCA para obtener un grupo comparativo respecto a las escalas aplicadas, ni se incluyeron sujetos prepúberes, los datos presentados son válidos únicamente para la población estudiada o para poblaciones con características similares.

En conclusión, los resultados del presente trabajo resaltan la importancia de identificar adolescentes en riesgo moderado de CAR con características prodrómicas de TCA, como la AU y la IC, las cuales cuando se presentan conjuntamente pueden mediar un efecto negativo sobre las CAR.

Se sugiere que en investigaciones futuras se considere validar el punto de corte utilizado en el presente trabajo, aplicando entrevistas que confirmen la evaluación realizada mediante el cuestionario de autorreporte. También se recomienda ampliar la población estudiada a prepúberes y varones para identificar en estos grupos el comportamiento de este punto de corte, así como efectuar un estudio longitudinal en jóvenes con sobrepeso $u$ obesidad que permita valorar situaciones de riesgo.

Agradecimientos. Las autoras desean agradecer en primer lugar a las alumnas que participaron en esta investigación. También expresan su gratitud a Francisco Juárez García, del Instituto Nacional de Psiquiatría "Ramón de la Fuente Muñiz", y a Alfredo Celis, profesorinvestigador de la Universidad de Guadalajara, por su apoyo en la realización de los análisis estadísticos, así como a Nadia González Briseño, pasante de la licenciatura en nutrición de la misma universidad, por su colaboración en la revisión bibliográfica del documento. Agradecen asimismo a Brígida Botello, por la corrección de estilo realizada al manuscrito, así como a Rosa Martha Meda Lara, por su asesoramiento como coordinadora de maestría en la Universidad de Guadalajara, dado que esta investigación fue parte de la tesis de grado presentada en esa universidad por una de las autoras. 


\section{REFERENCIAS}

1. Unikel Santoncini C, Bojórquez Chapela I, Carreño García S. Validación de un cuestionario breve para medir conductas alimentarias de riesgo. Salud Publica Mex. 2004; 46(6):509-15.

2. Gleaves DH, Brown JD, Warren CS. The continuity/discontinuity models of eating disorders - A review of the literature and implications for assessment, treatment, and prevention. Behav Modif. 2004;28(6):739-62.

3. Hunot C, Vizmanos B, Vazquez-Garibay EM, Celis AJ. Definición conceptual de las alteraciones de la conducta alimentaria. Disponible en: http://www.respyn.uanl. $\mathrm{mx} / \mathrm{ix} / 1$ /ensayos/alteraciones.htm Acceso el 11 de julio de 2011.

4. American Dietetic Association. Position of the American Dietetic Association: Nutrition intervention in the treatment of anorexia nervosa, bulimia nervosa, and other eating disorders. J Am Diet Assoc. 2006;106(12):2073-82.

5. Chamay-Weber C, Narring F, Michaud PA. Partial eating disorders among adolescents: A review. J Adolesc Health. 2005;37(5):417-27.

6. Sepulveda A, Carrobles JA, Gandarillas AM. Associated Factors of Unhealthy Eating Patterns among Spanish University Students by Gender. Span J Psychol. 2010;13(1):364-75.

7. Klump KL, Suisman JL, Burt SA, McGue M, Iacono WG. Genetic and environmental influences on disordered eating: An adoption study. J Abnorm Psychol. 2009;118(4): 797-805.

8. Field AE, Javaras KM, Aneja P, Kitos N, Camargo CA Jr, Taylor CB, et al. Family, peer, and media predictors of becoming eating disordered. Arch Pediatr Adolesc Med. 2008; 162(6):574-9.

9. Herzog DB, Eddy KT. Eating Disorders: What Are the Risks? J Am Acad Child Adolesc Psychiatry. 2009;48(8):782-3.

10. Grave RD. Eating Disorders: Progress and Challenges. European Journal of Internal Medicine. 2011;22(2):153-60.

11. Pinheiro AP, Sullivan PF, Bacaltchuck J, Prado-Lima PA, Bulik CM. Genetics in eating disorders: extending the boundaries of research. Rev Bras Psiquiatr. 2006;28(3):218-25.

12. Jacobi C. Psychosocial risk factors for eating disorders. In: Wonderlich S, Mitchell J, De Zwaan M, Steiger H, eds. Eating Disorders Review Part 1. United Kingdom: Radcliffe publishing; 2005. Pp. 15-172.

13. Polivy J, Herman CP. Causes of eating disorders. Annu Rev Psychol. 2002;53:187-213.

14. Krones PG, Stice E, Batres C, Orjada K. In vivo social comparison to a thin-ideal peer promotes body dissatisfaction: a randomized experiment. Int J Eat Disord. 2005;38(2): $134-42$.

15. Presnell K, Bearman SK, Stice E. Risk factors for body dissatisfaction in adolescent boys and girls: a prospective study. Int J Eat Disord. 2004;36(4):389-401.

16. Heatherton TF, Wyland C. Why Do People Have Self-Esteem? Psychol Inq. 2003;14(1):38.

17. O'Dea JA. School-based health education strategies for the improvement of body image and prevention of eating problems. An overview of safe and successful interventions. Health Education. 2005;105(1):11-33.
18. Instituto Nacional para el Federalismo y el Desarrollo Municipal (INAFED), México. Sistema Nacional de Información Municipal (SNIM). 7.a ed. México: INAFED; 2008.

19. Tuirán R, Fuentes C, Ávila JL. Índices de intensidad migratoria: México-Estados Unidos. México, D.F.: Consejo Nacional de Población, 2000.

20. Unikel Santoncini C, Gómez-Peresmitré G, Bojorquez-Chapela I. Manual de aplicación del cuestionario de factores de riesgo de trastornos de la conducta alimentaria [Informe interno]. México, D.F.: Instituto Nacional de Psiquiatría Ramón de la Fuente Muñiz; 2008.

21. Pope AW, McHale SM, Craighead WE, Garriga N. Mejora de la autoestima: Técnicas para niños y adolescentes. Barcelona: Editorial Martínez Roca; 1998.

22. Caso Niebla J. Validación de un instrumento de autoestima para niños y adolescentes [tesis de maestría]. México, D.F.: Universidad Nacional Autónoma de Mexico; 1999.

23. Unikel Santoncini C, Gómez Peresmitré G. Validez de constructo de un instrumento para la detección de factores de riesgo en los trastornos de la conducta alimentaria en mujeres mexicanas. Salud Mental. 2004;27(1): 38-49.

24. Stunkard AJ, Sorenson T, Schlusinger F. Use of the Danish adoption register for the study of obesity and thinness. In: Kety S, Rowland LP, Sidman RL, Matthyysse SW, eds. The genetics of neurological and psychiatric disorders. New York: Raven Press; 1983.

25. Acosta García MV, Gómez Peresmitré G. Insatisfacción corporal y seguimiento de dieta: una comparación transcultural entre adolescentes de España y México. Int J Clin Health Psychol. 2003;3(1):9-21.

26. Thompson JK, Altabe MN. Psychometric Qualities of the Figure Rating Scale. Int J Eat Disord. 1991;10(5):615-9.

27. American Psyquiatric Association. Diagnostic and Statistical Manual of Mental Disorders. 4th ed. Washington, D.C: American Psychiatric Association; 1994.

28. Unikel Santoncini C, Villatoro Velázquez JA, Medina-Mora Icaza ME, Fleiz Bautista C, Alcántara Molinar E, Hernández Rosario SA. Conductas alimentarias de riesgo en adolescentes mexicanos. Datos en población estudiantil del Distrito Federal. Rev Invest Clin. 2000;52(2):140-7.

29. Cole TJ, Bellizzi MC, Flegal KM, Dietz WH. Establishing a standard definition for child overweight and obesity worldwide: international survey. BMJ. 2000;320(7244):1240-3.

30. Griffiths JA, McCabe MP. The influence of significant others on disordered eating and body dissatisfaction among early adolescent girls. Eur Eat Disord Rev. 2000;8(4):301-14.

31. Dunkley TL, Wertheim EH, Paxton SJ. Examination of a model of multiple sociocultural influences on adolescent girls' body dissatisfaction and dietary restraint. Adolescence. 2001;36(142):265-79.

32. Amaya-Hernández A, Alvarez-Rayón GL, Mancilla-Díaz JM. Insatisfacción corporal en interacción con autoestima, influencia de pares y dieta restrictiva: Una revisión. Rev Mex Trastor Aliment. 2010;1(1):76-89.
33. Moreno González MA, Ortiz Viveros GR. Trastorno alimentario y su relación con la imagen corporal y la autoestima en adolescentes. Terapia Psicológica. 2009;27(2):181-9. Disponible en: http://redalyc.uaemex.mx/ src/inicio/ArtPdfRed.jsp?iCve=78511847004 Acceso el 12 de julio de 2011.

34. Canadian Paediatric Society. Dieting in adolescence. Paediatr Child Health. 2004;9(7): 487-91.

35. Borda Mas M, Avargues Navarro ML, López Jiménez AM, Torres Pérez I, Del Río Sánchez C, Pérez San Gregorio MA. Personality traits and eating disorders: Mediating effects of self-esteem and perfectionism. Int J Clin Health Psychol. 2011;11(2):205-27.

36. Cervera S, Lahortiga F, Martínez-González MA, Gual P, de Irala-Estévez J, Alonso Y. Neuroticism and low self-esteem as risk factors for incident eating disorders in a prospective cohort study. Int J Eat Disord. 2003;33(3):271-80.

37. Shaw HE, Stice E, Springer DW. Perfectionism, body dissatisfaction, and self-esteem in predicting bulimic symptomatology: lack of replication. Int J Eat Disord. 2004;36(1):41-7.

38. Peck LD, Lightsey Jr OR. The Eating Disorders Continuum, Self-Esteem, and Perfectionism. J Couns Dev. 2008;86(2):184-92.

39. Wiseman CV, Peltzman B, Halmi KA, Sunday SR. Risk factors for eating disorders: surprising similarities between middle school boys and girls. Eat Disord. 2004;12(4):315-20.

40. Gilbert N, Meyer C. Fear of negative evaluation and the development of eating psychopathology: a longitudinal study among nonclinical women. Int J Eat Disord. 2005; 37(4):307-12.

41. Sassaroli S, Ruggiero GM. The role of stress in the association between low self-esteem, perfectionism, and worry, and eating disorders. Int J Eat Disord. 2005;37(2):135-41

42. Garner DM, Garfinkel PE. The Eating Attitudes Test: An Index of the Symptoms of Anorexia Nervosa. Psychol Med. 1979;9(2): 273-9.

43. Olaiz-Fernández G, Rivera-Dommarco J, Shamah-Levy T, Rojas R, VillalpandoHernández S, Hernández-Avila M, et al. Encuesta Nacional de Salud y Nutrición 2006. Cuernavaca, México: Instituto Nacional de Salud Pública. 2006.

44. Bartsch AJ, Brummerhoff A, Greil H, Neumarker KJ. Shall the anthropometry of physique cast new light on the diagnoses and treatment of eating disorders? Eur Child Adolesc Psychiatry. 2003;12 (1 supl):I54-64.

45. Butryn ML, Wadden TA. Treatment of overweight in children and adolescents: does dieting increase the risk of eating disorders? Int J Eat Disord. 2005;37(4):285-93.

46. Lesar MD, Arnow B, Stice E, Agras WS. Private high school students are at risk for bulimic pathology. Eat Disord. 2001;9(2):125-39.

Manuscrito recibido el 19 de octubre de 2010. Aceptado para publicación, tras revisión, el 14 de junio de 2011. 
ABSTRACT Objective. Determine the relationship between the variables of self-esteem (SE), body dissatisfaction (BD), and body mass index (BMI) in a continuum of risky eating behaviors (REB) that ranges from normal behavior to the risk of eating disorders (ED),

\section{Continuum of risky eating behaviors in Mexican adolescents} thus facilitating early detection of adolescents with ED symptomatology and the degree to which such variables have an influence.

Methods. A total of 1982 young women aged 15-19 were selected through stratified random sampling. Self-esteem (Pope, McHale, and Craighead scale), body dissatisfaction (Stunkard's Figure Rating Scale), and body mass index were measured. A brief REB questionnaire was administered. Three categories of REB were defined: no risk, moderate risk, and high risk. A multiple regression analysis was performed.

Results. A direct relationship was found between risk of REB and BD, with significant differences between the groups $(P<0.001)$ : no risk ( $83.6 \%$ of sample), $54.1 \%$ had $\mathrm{BD}$; moderate risk (11.9\% of sample), $84.8 \%$ had BD; and high risk ( $4.5 \%$ of sample), $89.9 \%$ had BD. There were significant differences in SE and BMI only between the group with no risk and each of the risk groups. Self-esteem played a role in predicting REB in all the regression models evaluated, whereas BMI did not.

Conclusions. It is essential to identify adolescents at moderate risk of REB who have prodromic features of ED such as low SE and BD. When these features occur simultaneously, they can mediate a negative effect of BMI on REB.

Key words Eating behavior; adolescent health; self esteem; body mass index; body image; risk factors; obesity; overweight; Mexico. 